\title{
Ontology-based Function Optimization of S2B e-commerce Network Platform for College Students' Creative Works
}

\author{
Yang Caixia ${ }^{1,2}$, Chen $\mathrm{Wei}^{1}$ and Wei Duo ${ }^{2}$ \\ 1 School of Economics and Management, Harbin Engineering University, Harbin \\ , China; \\ 2 School of Management, Harbin University of Science and Technology, Harbin, \\ China \\ yangcaixia666@163.com,weichen666@126.com,249093790@qq.com
}

\begin{abstract}
Student to business(Abbr. S2B) e-commerce network platform is designed to display the college students' creative works, and build joint channel for the creative talents of college and small and medium-sized enterprises requiring innovative resources. Firstly, this paper analyses the targets and features of S2B e-commerce platform. Secondly, the user requirements-oriented explicit functional demands are listed from two aspects of the individual users and enterprise users through investigation. In addition, implicit functional demand is obtained based on ontology. According to the method of ontology mapping, the function description by natural language is mapped to semantic elements and demand items, then the relationship of demand items coming into semantic structures. According to the method of ontology inference, the semantic structure is mapped to domain ontology of S2B e-commerce network platform, as well as the implicit functional requirements of platform are digging out. As a result, the whole functional structure tree of $S 2 B$ e-commerce network platform is constructed. Finally, the detail functions of the platform are described by UML modeling tool. Through the platform the college students' creative works are integrated and flow to meet the innovative demand of enterprises. $S 2 B$ e-commerce model can make the creative resources be fully used and put forward the innovative and creative industry developing well.
\end{abstract}

Keywords: S2Be-commerce, Network platform, Ontology, Creative industry

\section{Introduction}

With the development of e-commerce and third-generation network technology continuing to expand, the traditional enterprise model can't adapt to the times and the new requirements. So the creative industries have emerged. Currently, many western countries began to gradually penetrate the creative thinking into the economic dimension. The knowledge as the core competitiveness is leading the modernization of the traditional enterprise model [1], and also creating a high degree of skill and fluidity of the creative industries.

However, in order to develop creative industries, creative talent is essential. The talent is a source of creativity. Compared with some better creative industries in the developed countries, the development of China in this area is still not mature enough. Enterprises are difficult to find creative talent and it results in a lack of creative talent.

In our universities, professionals involved with new ideas become more and more. Subsequently more a large number of students can create new ideas and new creative products, which certainly have some market value. But up to now there is not a dedicated and effective platform for them to show their creativity and creative works. So it is harder for enterprises to find these valuable ideas and works. The S2B e-commerce model for 
the innovative and creative industries is proposed, and subsequently college S2B ecommerce website platform for the creative industries is ready to be built as the bridge between the college students and the enterprises to present and exchange information. On this platform for the students, the creative talent can not only demonstrate their creativity and creative works, but also can easily communicate with each other, which can help generate ideas collision and the creative spark. On the other hand, for the enterprises, they can browse their favorite creative works and directly communicate with the publisher of the creative works. Furthermore, if enterprises and publishers have cooperative intention, they can even sign a cooperation agreement to recruit contract. Companies can also publish some of the requirements on the platform, some members to complete the task of bonuses, either to reduce enterprise costs, they can get a better product earlier contact with the community to help students understand the enterprise needs to a certain extent, more Good to enhance their knowledge and skills faster.

\section{The Targets and Features of S2B e-commerce Network Platform}

The research object of this paper is S2B e-commerce network platform, which is to provide an information platform to display, cooperate and exchange for the students of the college and enterprises. The S2B e-commerce network platform can increase the matching degree between the demand of enterprise and the supply of creative talent or works of college students. It can achieve more effective use of creative resources. The $\mathrm{S} 2 \mathrm{~B}$ e-commerce model is shown in figure 1.

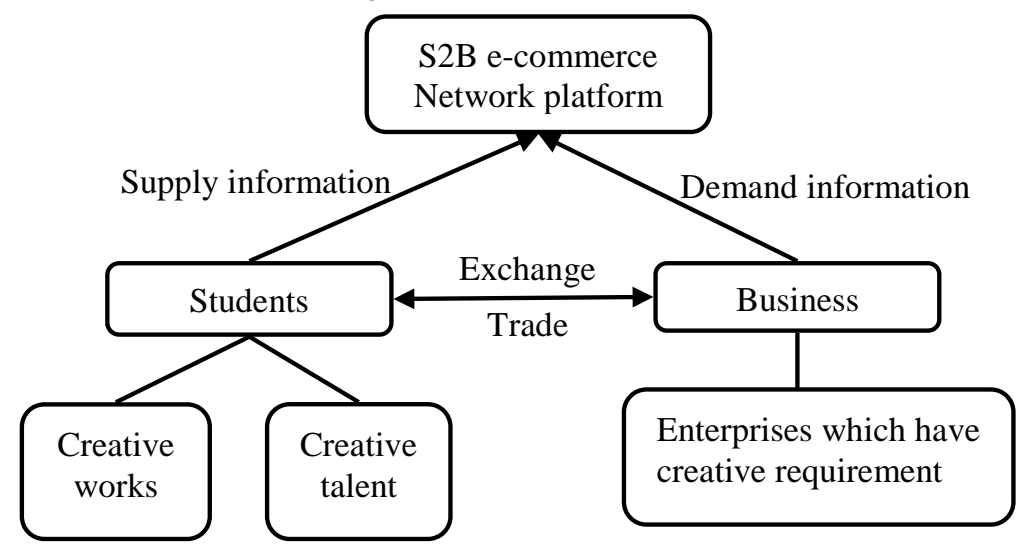

Figure1. S2B e-commerce Model

\section{1. The Targets of S2B e-commerce Network Platform}

i) Provide a platform for enterprises user to release their own demand information and search personnel resources through enterprises user management module.

ii) Provide more opportunities for college students to present themselves and make more valuable creative talent to meet the enterprise needs through personal user management module.

iii) Build a platform for the free exchange and trading between enterprises and individuals by the data supporting web.

iv) Build an e-commerce network platform management system with high accountability and flexibility.

v) Establish a high-performance, secure and reliable database systems, which include information collection, processing, backup and restore functions, the reliable and efficient types of database entities.

vi) Take advantage of cloud innovation model to promote the aggregation of resources and innovation to achieve rapid development of creative industries. 
vii) In the context of the creative industries to promote the realization and development of S2B e-commerce models, and make the S2B e-commerce model reasonable operation.

\section{2.The Features of S2B e-commerce Network Platform}

i) From the real needs of the creative industry development, put forward S2B ecommerce model for college students to help develop innovative thinking of contemporary college students, who need a high performance display platform to display their innovative ideas and creative works .

ii) The S2B e-commerce network platform belongs to a type of innovative website, and its functional demand is complex.

iii) Interacting through space, forums and other means, the user will get a lot of demand and supply information in time.

iv) Clear content classification and rich type of information can be passed and communicated. We can create more innovative resources from the perspective of cloud innovation, and make the innovation resources to obtain the reasonable effective use.

\section{Ontology-based Function Optimization of Platform}

Aiming at the problems such as fuzziness of the requirement, professional limitations, nature language of the expression in the process of development on network platform, a method for the implicit requirements elicitation of the network platform based on ontology was presented. The foundation function module domain ontology and rules of inference methods for extensions and modifications mapping function form a more perfect new needs analysis. UML modeling tools were used for system analysis and design.

According to the targets and features of S2B e-commerce platform, the user requirements-oriented explicit functional demands are listed from two aspects of the individual users and enterprise users through investigation. In addition, implicit functional demand is obtained based on ontology. According to the method of ontology mapping, the function description by natural language is mapped to semantic elements and demand items, then the relationship of demand items coming into semantic structures. According to the method of ontology inference, the semantic structure is mapped to domain ontology of S2B e-commerce network platform, as well as the implicit functional requirements of platform are digging out. As a result, the whole functional structure tree of S2B e-commerce network platform is constructed. The detail functions of the platform are described by UML modeling tool. Finally, the basic function is developed by Asp.net. Through the platform the college students' creative works are integrated and flow to meet the innovative demand of enterprises.

\subsection{User requirements-oriented Explicit Requirements Investigation of Platform}

Since the purpose of S2B e-commerce network platform is designed to build an effective exchange platform among college students and enterprise. In order to make the design of the platform capabilities closer to the user needs, we conducted a market survey of platform functional requirements among the relevant population [2]. Extracted from the survey results of the analysis, the user's initial requirements are summarized and expressed as follows.

The initial functional requirements of the individual users:

i) The needs of self-presentation for individual users.

ii) The needs of seeking information sharing of individual users.

iii) The needs of seeking self-improvement for practice experience.

iv) The needs of seeking more and better jobs information from enterprises.

The initial functional requirements of enterprises users: 
i) The needs of issuing the non-core tasks for enterprises.

ii) The needs of forming an effective link with the college students.

iii) The needs of searching for innovative talents from universities.

According to the initial user requirements sorted out above, the platform functional requirements can be broken down as follows.

For the individual users: the individual user's self-presentation function requires a clear and powerful platform for the individual users to upload works and ensure a clear distinction between areas uploaded and the corresponding fields exhibition. The visitors can browse works in the exhibition, post comments and download it with the works exchange. Individual users need an in-time exchange forum. The forum users can not only find fellow person and exchange knowledge sharing, but also be able to find relevant tasks or employment opportunities posted by other users or enterprises. Through good ability to complete tasks, college students can achieve self-improvement and better understanding of the current status and demand of the development of enterprise professionals. It's helpful for them to lay a solid foundation for future employment. In order to enable users to upload works and publish other relevant information more flexibility, users need a personal comprehensive information storage space for the information storage and management.

For the enterprise users: some non-core tasks can be published to the platform. The enterprises can select the appropriate student to sign the project agreement and complete the tasks together. Enterprises can upload the task information for individual users to browse and consult. You can find much better creative works to tap its potential enterprise opportunities. Meanwhile some potential talent also can be absorbed into the enterprise. In the forum enterprises also can publish job information and the user can select a trusted person strong purpose in the transaction over the individual user in recruitment. Similar to individual users, in order to better define user roles and manage the publishing of information, enterprise users also need a space to facilitate enterprise management for the publishing of information while allowing more users to understand the supply and demand situation of enterprises. So as a corporate user can build more mutual channels.

\subsection{Ontology-based Implicit Requirements Elicitation for Platform}

The requirement analysis of the S2B e-commerce network platform has the characteristics of ambiguity and uncertainty. So in order to reduce ambiguity and uncertainty, and reason out some potential system function, the ontology-based method to information system requirements elicitation is put forward to optimize the function of S2B e-commerce network platform. Firstly, the natural language system has described some basic functions, and these basic functions should have been mapped into semantic elements. Then the semantic elements have been combined to express the demand entries [3]. According to the relationship between the entries listed and the semantic ontology structure, the relationships between concepts have been analyzed and defined. According to the semantic structure by mapping entry, the domain ontology of the S2B e-commerce network platform has been mapped. At last, the implicit requirements can be found according to the ontology reasoning rules. The implicit requirements obtained through inference rules can improve the functionality and the effectiveness of functional requirements analysis of the whole network platform [4].

The S2B e-commerce network platform for college students' creative works has many functional requirements. Only some initial requirements are selected and analyzed here. Ontology simplified flowchart of S2Be-commerce network platform is shown in Figure 2: 


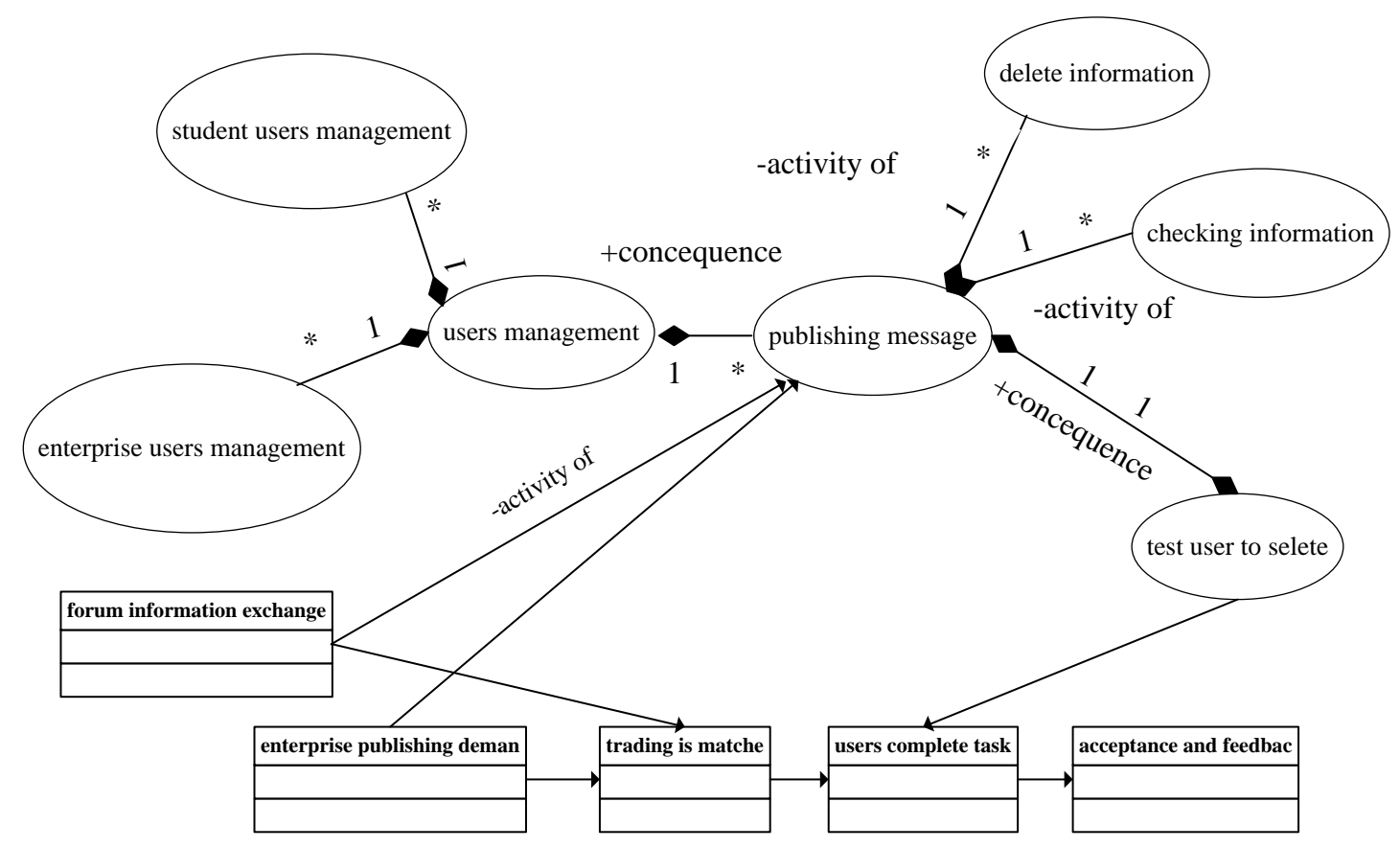

\section{Figure 2. Ontology Simplified Flowchart of S2Be-commerce Network Platform}

Matching supply and demand graph user management class e-commerce network platform to achieve the S2B registered user management, providing services and to set appropriate permissions for different types of users, enterprise users and individual users in demand management task management classes effective functioning of the chain. User management class is a binary relation Constraint-of the range, the demand constraint management tasks[5].

Household information released by the platform for review, modification, by other operations, to ensure the legitimate needs of the validity of the information published by users, so that the user's transaction sides in a safe, efficient and legitimate on the basis of the information published is Part-of, Activity-of the range, is a constraint Constraint-of subordinate objects.

In order to define the system requirements much clearer and accurate statement, this article will list the platform requirements to be stated by the ontology semantic mapping. The demand list depose of S2B e-commerce network platform and the ontology mapping process from demand list are shown in figure 3. Some partial list of requirements and their interaction ontology mapping examples are shown in figure 4.

The mapping process[6] is described and analyzed in detail as follows:

\# 1 User published information can be related operations

\# 1.1 Enterprise users, publish the information one needs, the cost of this section is mapped body element "enterprise users" and "Wanted" By mapping entry \#. And demand entry \# is also mapped to "apply" relationship between "enterprise users" and "demand information". Concept mapping is Actor (enterprises). 


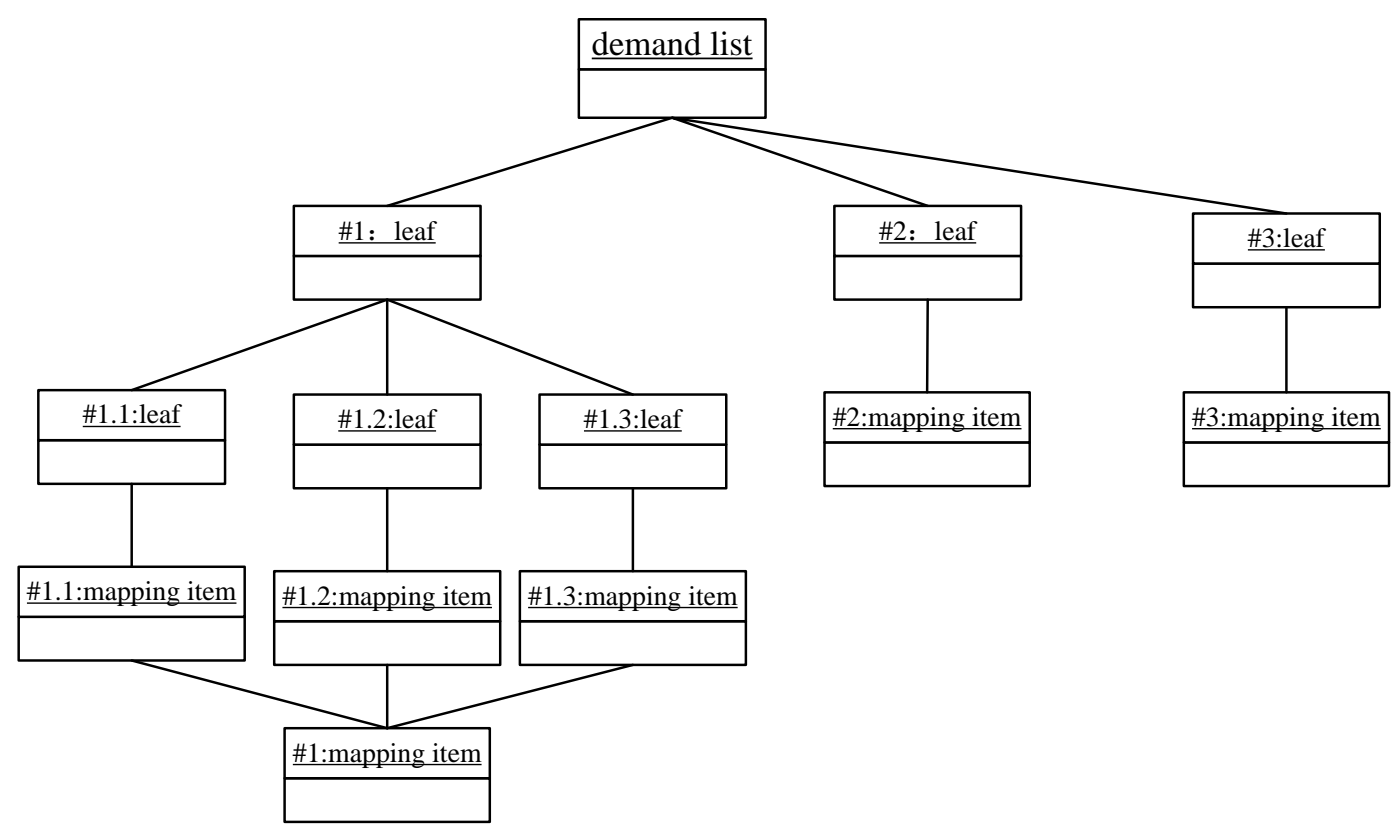

Figure 3. The Ontology Mapping Process from Demand List

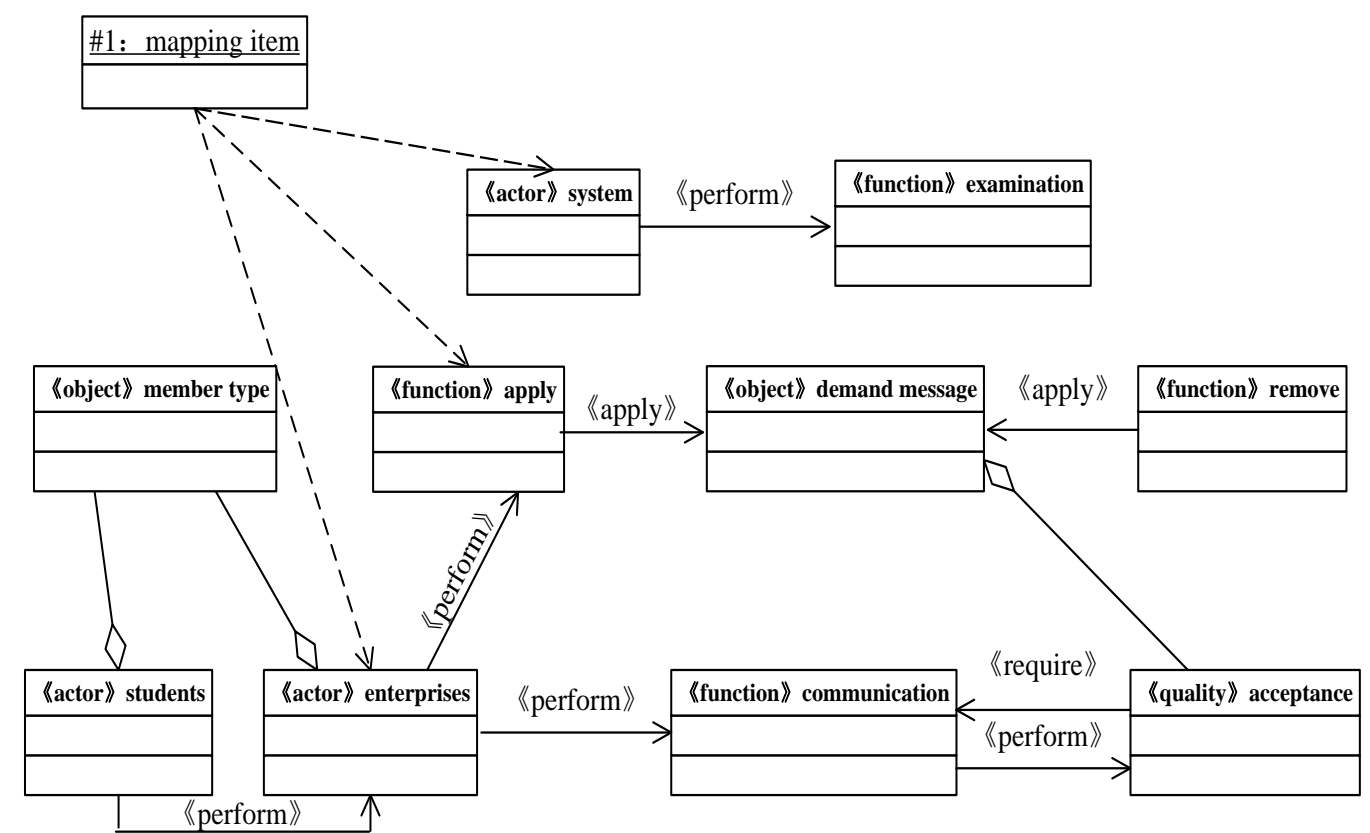

Figure 4. Partial List of Requirements and their Interaction Ontology Mappings

\# 1.2 systems management tasks demand release of an audit by the entry \# 1, the cost of entry to map the body element "system administrator" and "audit." And demand entry \# is mapped to perform relationship between "system administrator" and "audits". Mapping concept that is function (examination) and object (demand message).

\# 2 can be carried out between enterprise users and individual users exchange and communication, by mapping entry \# 2 , the cost of this section is mapped body element 
"enterprise users" and "communication." And demand entry \# is also mapped to "perform" relationship between "enterprise users" and "communication". Concept mapping is a function (communication) and Actor (enterprises).

\# 3 information published by users needing to be timely processed

\# 3.1 release was reviewed by the need for timely entry \# 3, the cost of this section is mapped body element "Wanted" and "audit." And demand entry \# is mapped to "demand information" and "audit" require the relationship between the concept mapping is a function (examination ) and object (demand message).

\# 3.2 Remove demand information due to the demand for information is matched by individual users and removed 3, the cost of this section is mapped body element "Wanted" and "acceptance" by mapping entry \#.

\subsection{The Whole Functional Structure of Platform}

Based on the above mapping elements that can clarify its meaning in the demand for college entry S2B e-commerce network platform body, and finally we can get a list of requirements to expand and improve the body through the use of inference rules set.

As shown by the expression of ontology reasoning function demand list to demand tree, a requirements tree is composed of the initial demand information by ontology reasoning after pruning, add to the demand information. The whole system structure tree of platform is shown is figure 5 .

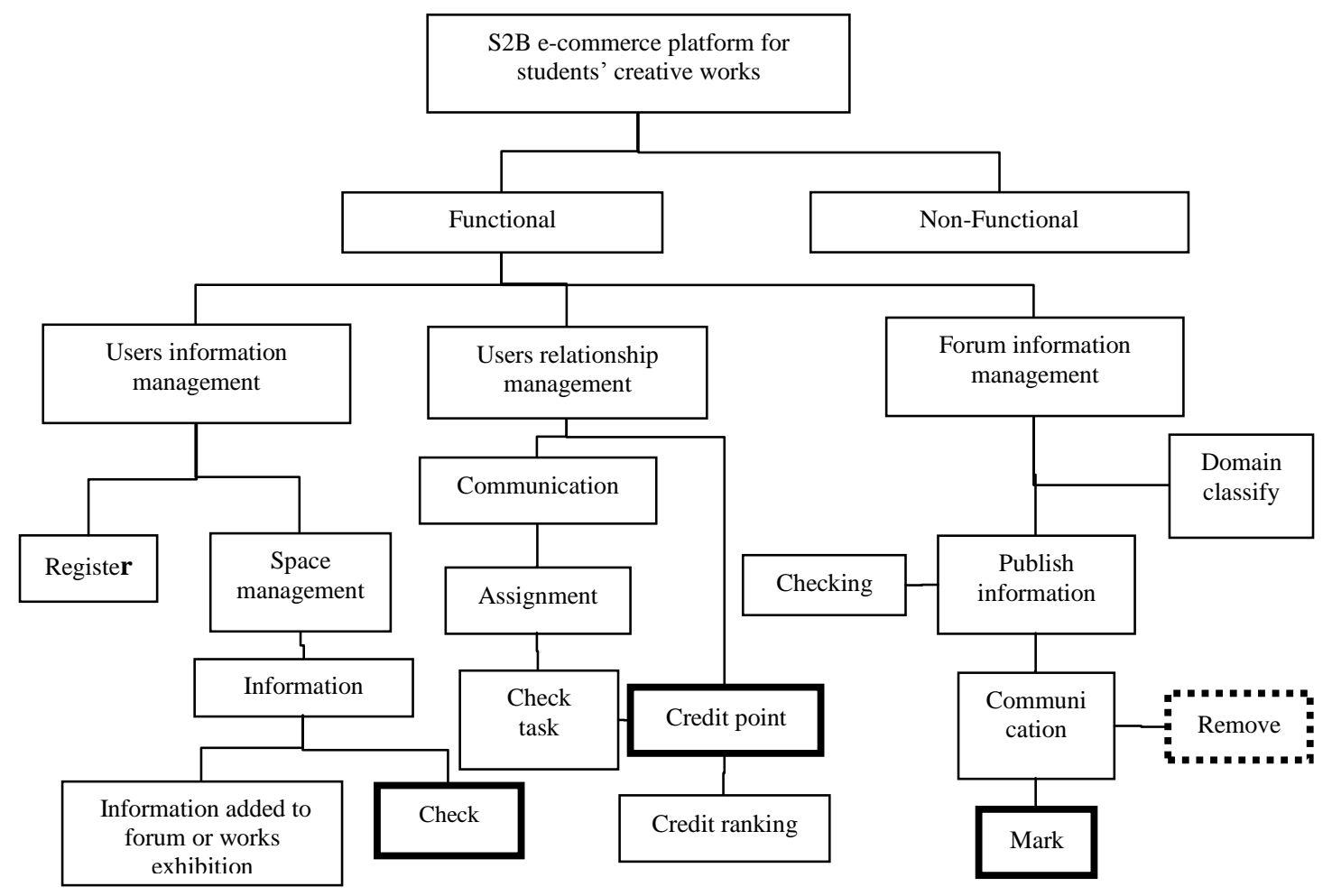

Figure 5. The Structure Tree of Platform

In Figure 5, the function of S2B e-commerce network platform is optimized by the ontology reasoning method. The initial function in the dashed boxes need to be removed after the function reasoning. Meanwhile the function in the bold black boxes represents the inference drawn increasing demand for new features. Release the user information into effect through approval system audit in the forum or works published before. On completion of the transaction and the user acceptance through demand-side tasks. Task 
evaluation information will be fed directly into the user's credit score.The task can also be performed by integration into the user's rankings. The published information in forum will be removed after expiration $t$ in order to ensure the validity of the information and ready to check the integrity. The outdated information can be saved and marked as expired [7].

\section{Design and Implementation of the Function for Platform}

The use case diagram in UML modeling tools[8] are used to describe the basic functions of the platform. The use case diagram of S2B e-commerce network platform is shown as Figure 6.

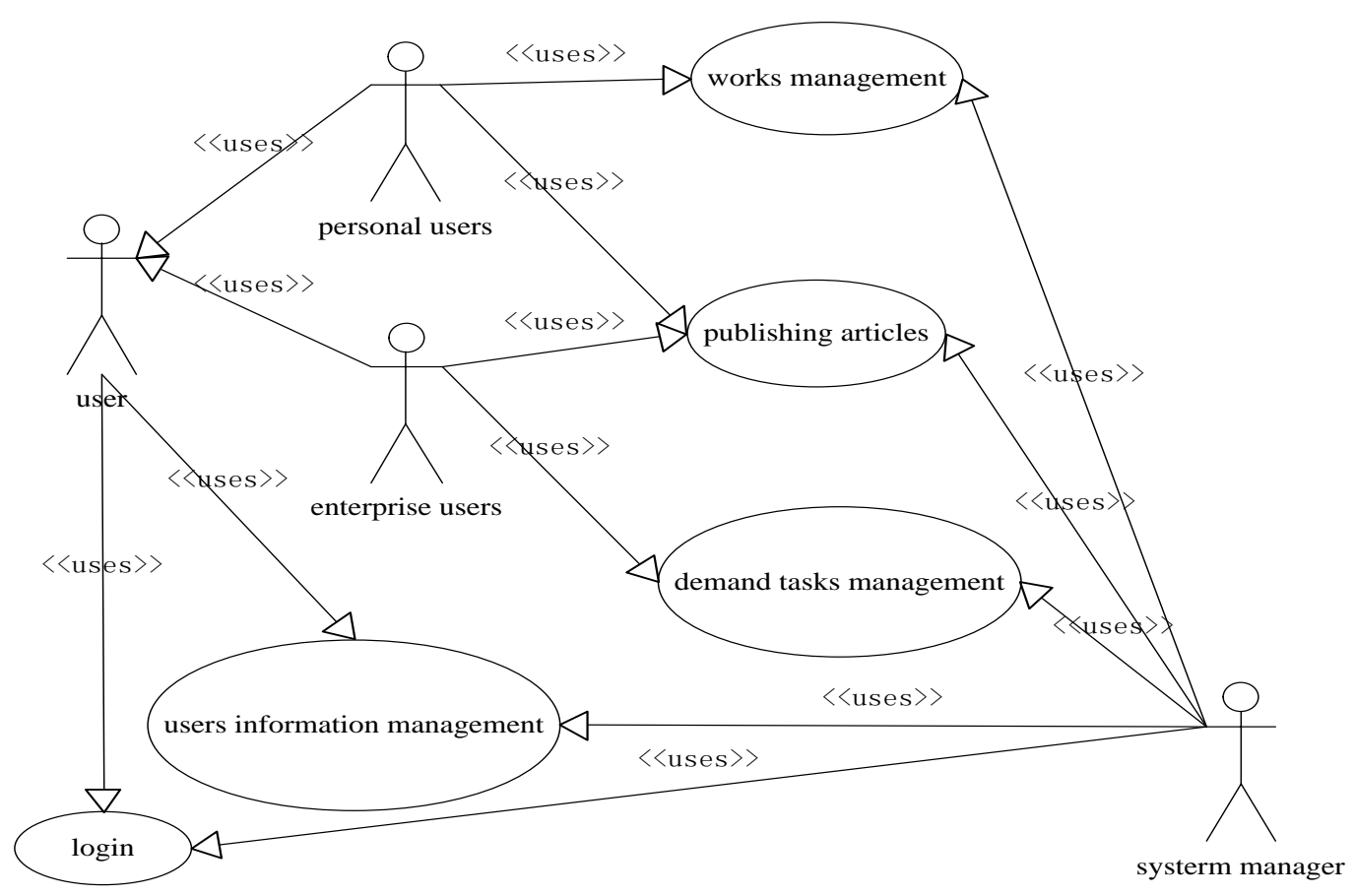

Figure 6. The Use Case Diagram of S2B e-commerce Network Platform

Firstly, the needs of platform are analyzed. And then the corresponding operations are decomposed to identify and find the major business of process in enterprises. The use case in UML is responsible for the business or process. Secondly, the corresponding use case diagram can be drawn by the UML modeling language, which should indicate that each participant is responsible for the use cases and relationship between the various participants. The detailed description of each use case should be given. For example, according to the role of division administrators, personal users, enterprise users case diagram and corresponding portions were treated with descriptions, as well as in accordance with the registered enterprise division, publish the works, publishing needs task and published the use case diagram of the article and some use case description.

The basic functions of S2B e-commerce network platform are developed by Asp.net. Through the platform the college students' creative works are integrated and flow to meet the innovative demand of enterprises. The home page is shown as figure 7. 


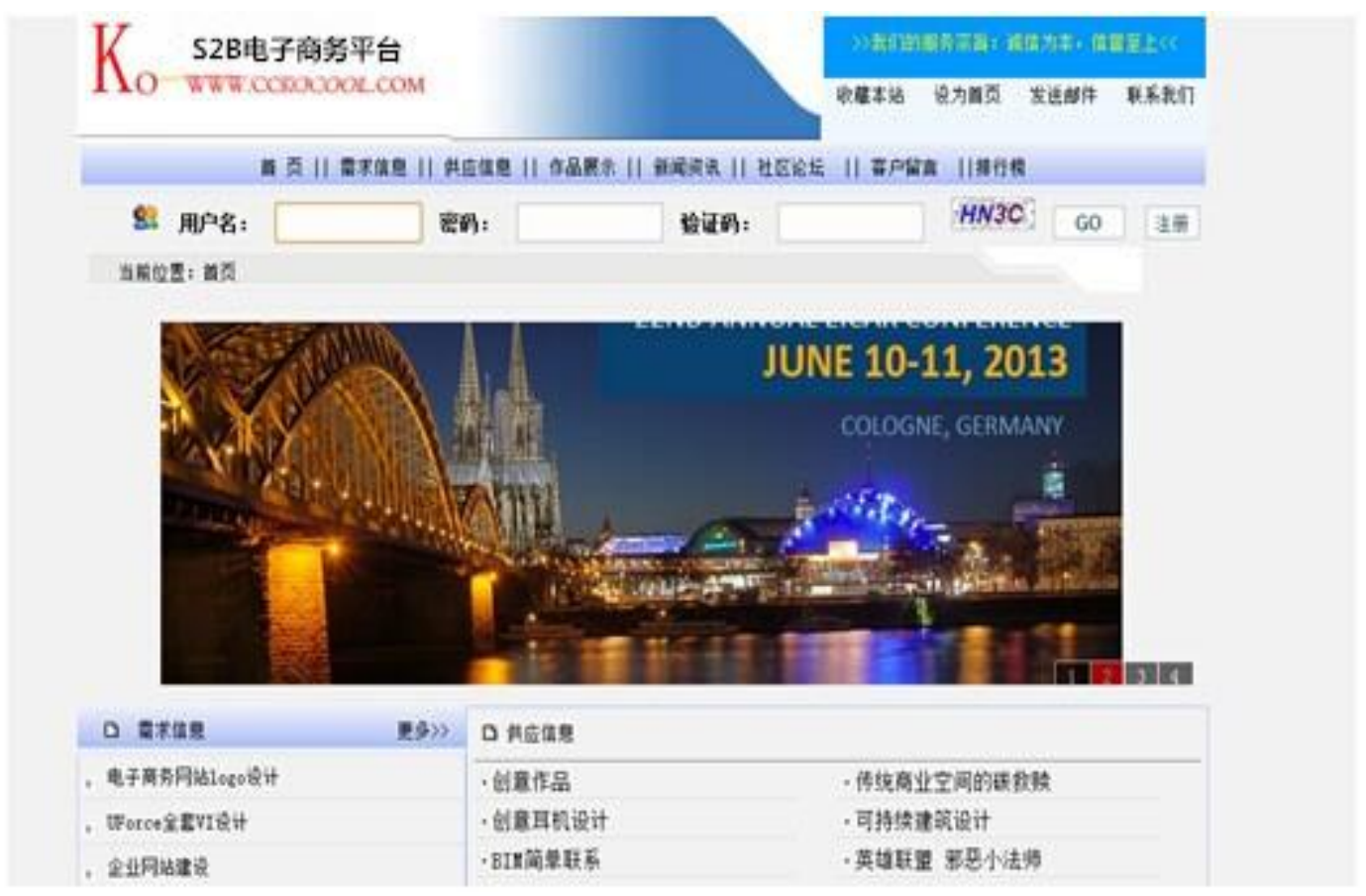

Figure7. The Home Page of S2B e-commerce Network Platform

\section{Conclusions}

The development of the innovative and creative industry is becoming more and more important. The S2B e-commerce mode is a helpful style for the combination between the creative industry and e-commerce. The targets and features of S2B e-commerce network platform for the college students' creative works are introduces. From the aspect of service oriented the functional requirement and information flow among platform users are analyzed. From the viewpoint of user demand attribute, the ontology mapping method and relevant reasoning method are applied to design and optimize the whole framework of S2B e-commerce network platform is designed and optimized, which include the functional requirements, non-functional requirements and other aspects. Finally, the detail functions of the platform are described by UML modeling tool. Through the platform the college students' creative works are integrated and flow to meet the innovative demand of enterprises. S2B e-commerce model can make the creative resources be fully used and put forward the innovative and creative industry developing well.

\section{Acknowledgement}

The research work was supported by Undergraduate Training Programs for Innovation and Entrepreneur of Heilongjiang Province "The operation of C2B e-commerce platform for College Students' Creative Works", Chinese National Natural Science Foundation under Grant No. 71272191, and Postdoctoral Foundation of Heilongjiang Province under Grant No. LBH-Z09151. 


\section{References}

[1] Q. F. Wang and M. H. Shi, "China's cultural and creative industry cluster innovation mechanisms and countermeasures", Proceedings of International Academic Conference of Art Engineering and Creative Industry, (2012); Fuzhou, China.

[2] W. B. Dong, "On the software requirements analysis", Science \& Technology Vision, no. 26, (2012), pp. 236-237.

[3] L. L. Zhai, T. Zhang and D. H. Peng, "Ontology-based requirements elicitation of information system", Computer Integrated Manufacturing System, vol. 19, no. 1, (2013), pp. 173-180.

[4] X. G. Wang, Y. L. Li and H. Han, "Ontology-based sharing system design of software requirements", Journal of Lanzhou Jiaotong University, vol. 28, no. 4, (2009), pp. 82-85.

[5] C. L. Yan and X. Q. Tang, "Ontology Modeling of E-commerce Domain Knowledge", Journal of Chongqing Three Gorges University, vol. 29, no. 3, (2013), pp.128-130.

[6] Y. P. Teng, Z. Z. Lian and H. Z. Wang, "A demand information ontology construction method based on semantic meta model”, Information Science, vol. 27, no. 11, (2009), pp.1695-1699.

[7] K. Wang, G. Y. Li and W. L. Zhang, "The information needs of the body for e-commerce", Computer and Digital Engineering, vol. 35, no. 5, (2007), pp. 124-127.

[8] Y. Tan and Z. F. Xie, “Analysis and design of the Portal for Private Enterprises Association”, Journal of Guangdong Polytechnic Normal University, no. 3, (2011), pp. 25-27. 